\title{
Magnetoencephalography in Preoperative Epileptic Foci Localization: Enlightenment from Cognitive Studies
}

\author{
Qian Wang ${ }^{1,2}$, Pengfei Teng ${ }^{2}$ and Guoming Luan 1, 2,3* \\ ${ }^{1}$ Beijing Key Laboratory of Epilepsy, Sanbo Brain Hospital, Capital Medical University, Beijing, China, ${ }^{2}$ Department of \\ Neurosurgery, Epilepsy Center, Sanbo Brain Hospital, Capital Medical University, Beijing, China, ${ }^{3}$ Beijing Institute for Brain \\ Disorders, Capital Medical University, Beijing, China
}

OPEN ACCESS

Edited by:

Yang Xia

University of Electronic Science and Technology of China, China

Reviewed by:

Honghui Zhang,

Northwestern Polytechnical University,

China

Qishao Lu,

Beihang University, China

*Correspondence:

Guoming Luan luangm3@163.com

Received: 15 May 2017 Accepted: 12 June 2017 Published: 28 June 2017

Citation:

Wang Q, Teng P and Luan G (2017)

Magnetoencephalography in

Preoperative Epileptic Foci Localization: Enlightenment from

Cognitive Studies.

Front. Comput. Neurosci. 11:58.

doi: 10.3389/fncom.2017.00058
Over 30\% epileptic patients are refractory to medication, who are amenable to neurosurgical treatment. Non-invasive brain imaging technologies including video-electroencephalogram (EEG), magnetic resonance imaging (MRI), and magnetoencephalography (MEG) are widely used in presurgical assessment of epileptic patients. This review mainly discussed the current development of clinical MEG imaging as a diagnose approach, and its correlations with the golden standard intracranial electroencephalogram (iEEG). More importantly, this review discussed the possible applications of functional networks in preoperative epileptic foci localization in future studies.

Keywords: magnetoencephalographic (MEG), intracranial electroencephalogram (iEEG), distributed source modeling (DSM), resting-state, functional connectivity

\section{INTRODUCTION}

Epilepsy is defined as a brain disorder characterized predominantly by recurrent and unpredictable interruptions of normal brain function resulting from abnormal and excessive neuronal discharges (Fisher et al., 2005). Approximately a third of epileptic patients are refractory to medication, who are amenable to neurosurgical treatment if evidences clarify focal network underlying the epilepsy (Jobst and Cascino, 2015). The main purpose of presurgery evaluation is to localize the epileptic foci in order to direct a precise surgery plan for an individual patient. Various of non-invasive brain imaging technologies including video-electroencephalogram (EEG), magnetic resonance imaging (MRI), positron emission tomography (PET), single photon emission computerized tomography (SPECT), and magnetoencephalography (MEG) play crucial roles in presurgical assessments of epileptic patients (Duncan et al., 2016). However, when the non-invasive outcomes are inconsistent, intracranial-electroencephalogram (iEEG), which is the current "golden standard" for localization of seizures, is needed to be conducted to confirm the involvement of suspected brain regions.

Among those non-invasive technologies, MEG has held the promise for localizing epileptic zones for its predictions of favorable post-surgical outcomes (Zhang et al., 2011; Englot et al., 2015b) and concordance with iEEG results (Almubarak et al., 2014; Grova et al., 2016; Murakami et al., 2016). One of the main advantages of MEG is its high spatial and temporal accuracy. By detecting the changes of magnetic fields produced by the electrical activities of neurons, epileptic spikes could be observed in MEG within only $4 \mathrm{~cm}^{2}$ cortical generators (Mikuni et al., 1997; Oishi et al., 2002; Shigeto et al., 2002). While a minimum area of $10 \mathrm{~cm}^{2}$ was required to detect spikes in scalp EEG (Tao et al., 2007). 
Although MEG has numerous advantages in epileptic foci detection, it still can't replace the iEEG as an independent modality in presurgical assessment (Duncan et al., 2016). The most common MEG application now is to guide implantation sites for intracranial recordings (Knowlton et al., 2009; Agirre-Arrizubieta et al., 2014). One of the most important limitations exist in clinical MEG studies is the immature source reconstructing algorithm which is still developing. In the past decade, both the quantity and quality of MEG studies have remarkable increased, this state of the art technique seems promising to reveal brain mechanism of cognitive processing (Baillet, 2017). Going deep into understanding of human's brain, a large number of new concepts and perspectives are emerging. How could we take advantage of the exciting progresses made in cognitive MEG studies? This review attempted to discuss the recent development of clinical MEG studies and its application in preoperative epileptic foci localization.

\section{SOURCE RECONSTRUCTION OF MEG DATA}

Interictal epileptic discharge (IED), which represents the field summation of potentials from a population of pathologically synchronized bursting neurons, is generally considered as a robust biomarker that identifies the epileptic foci (Oishi et al., 2002, 2006). However, the raw MEG signals are magnetic fields outside the human scalp (Cohen, 1968). A combination of MEG signals and structural MRI scans is needed to estimate the location and intensity of IEDs sources inside the brain which is called source localization. After artifact rejection and noise definition, source localization processing could be generally divided into two steps: (1) head modeling which establishes the electromagnetic properties of the sensor array and estimations of the brain sources; (2) source modeling imaging which solves an inverse problem (Tadel et al., 2011; Baillet, 2017).

Reconstructing of current dipoles, which are convenient model equivalents to the post-synaptic electrophysiological activity of local neuronal assemblies, is the basic concept in MEG source estimation process (Mosher et al., 1992). Among the current studies, two main approaches have been well adopted: single equivalent current dipoles (sECD), where the position and amplitude of one sECD is estimated over relatively short time windows, and distributed source modeling (DSM), which attempts to explain a given observed magnetic field by the distribution of source intensities in the brain instead of a single source (Tadel et al., 2011; Baillet, 2017).

The most common algorithm of the epileptic foci localization from clinical MEG recordings is sECD (Bagic et al., 2011). A well trained magnetoencephalographer is needed to extracts tightness and orientation of the dipole clusters visually (Salayev et al., 2006). Ideally, sECD can localize at best the center of the spatially extended generators as a point source (Ebersole, 1997). A retrospective study used sECD method revealed that patients with tight cluster and stable orientation dipole could significantly predict a better outcomes after surgery (Murakami et al., 2016).

However, the practicability of sECD method is debated not only for the subjective selection of IED period, but also for the possibility to mislead the presence of large spatially extended generators (Kobayashi et al., 2005). The latter is sophisticated because the IEDs were sometimes not confined to only one area (Agirre-Arrizubieta et al., 2009).

In the past decade, DSM has been largely promoted in cognitive MEG studies based on the fact that multiple brain areas would activate simulatonesly when a participant is performing a certain task (Tadel et al., 2011). DSM has also been applied in presurgical MEG evaluation using the nonlinear Maximum Entropy on the Mean (MEM) algorithm (Chowdhury et al., 2013; Grova et al., 2016; Heers et al., 2016; Zerouali et al., 2016), which could better predict the cortical regions with IEDs compared with other distributed models (Heers et al., 2016).

\section{CORRELATION WITH INTRACRANIAL DATA}

To evaluate the effectiveness of epileptic foci localization in MEG, the agreement between the MEG and "gold standard" invasive EEG approaches, which mainly include electrocorticography (ECoG) and stereo-electroencephalogram (sEEG) should be considered. Although most studies focus on the evaluation consistency between MEG and ECoG (Oishi et al., 2002, 2006; Knowlton et al., 2009; Almubarak et al., 2014), more and more studies has paid attention to the correlations between MEG and sEEG (Bouet et al., 2012; Grova et al., 2016; Murakami et al., 2016), which is considered to be minimal invasive and more suitable for preoperative evaluation (Gonzalez-Martinez et al., 2013; Zhou et al., 2017).

The effectiveness of MEG is depends on the depths of IED sources. Previous studies showed that MEG could detect $50-90 \%$ of spikes detected on iEEG superficial cortex including the lateral temporal cortex (Shigeto et al., 2002; Oishi et al., 2006; AgirreArrizubieta et al., 2009), while it could detect about 50-63\% iEEG spikes in mesial temporal structures (Santiuste et al., 2008). These results are in general acceptable for presurgical assessments. Recent studies have revealed that epileptic activity from the insula, which is far away from scalp, can also be detected by MEG (Heers et al., 2012; Mohamed et al., 2013).

The limitation of iEEG approaches is it can only monitor a portion of brain areas, while MEG can provide a broad view of whole head activities and therefore may aid the interpretation of iEEG data. Murakami et al. (2016) systematically examined the consistency between the interictal discharges in MEG with interictal and ictal discharges in sEEG in 50 retrospective cases. The results showed that the proportion of seizure-free after epileptic foci resection was significantly higher when sEEG completely sampled the area identified by MEG. This finding suggests that although the MEG is still unable to take the place of iEEG as the standard, more weight should be assigned to MEG outcomes in combination of multiple modalities in preoperative evaluation.

\section{EPILEPSY AS A NETWORK DISEASES}

One of the core questions in cognitive neuroscience is how different brain areas cooperate during perceptual/cognitive 
processing (Bressler and Menon, 2010). This hypothesis could also applied when epilepsy is considered as a network diseases (Laufs, 2012). Recent studies suggested that the brain connectivity differences among individuals could effectively interpret the patients with differing surgical outcomes (Englot et al., 2015a; Munsell et al., 2015).

Traditional epileptic network is defined as discharge propagation network (Malinowska et al., 2014), which is correlated with patient surgical outcome (Tanaka et al., 2014). However, a portion of patients could not be captured epileptic discharges during MEG recording. Functional connectivity studies have been recently applied in epileptic patients based on the assumption that the normal functions of local networks are impaired in the foci areas. Evidence for altered focal functional connectivity in patients with temporal lobe epilepsy has been reported using resting-state functional MRI method (Haneef et al., 2012; Cataldi et al., 2013; Maccotta et al., 2013).

Unlike the extensive studies using function MRI modality, the resting-state MEG studies are rare. Krishnan et al. (2015) conducted a preliminary MEG study and suggested that accurate localization of the epileptogenic foci may be accomplished using non-invasive spontaneous resting-state signals without the need to capture IEDs. Zerouali et al. (2016) used resting-state MEG analysis to recognize insular epileptic foci and revealed that anterior and posterior sub-regions of the insula have distinct patterns of functional connectivity, which could further guide different surgery plans.

A combination of resting-state fMRI and MEG studies of functional connectivity could be adopted to investigate the pathophysiology of epileptic networks. However, whether there is potential benefit in prediction of epilepsy surgery outcome in individual level is not established yet. To achieve this goal,

\section{REFERENCES}

Agirre-Arrizubieta, Z., Huiskamp, G. J. M., Ferrier, C. H., vanHuffelen, A. C., and Leijten, F. S. (2009). Interictal magnetoencephalography and the irritative zone in the electrocorticogram. Brain 132, 3060-3071. doi: 10.1093/brain/awp137

Agirre-Arrizubieta, Z., Thai, N. J., Valentín, A., Furlong, P. L., Seri, S., Selway, R. P., et al. (2014). The value of magnetoencephalography to guide electrode implantation in epilepsy. Brain Topogr. 27, 197-207. doi: 10.1007/s10548-013-0330-x

Almubarak, S., Alexopoulos, A., Von-Podewils, F., Wang, Z. I., Kakisaka, Y., Mosher, J. C., et al. (2014). The correlation of magnetoencephalography to intracranial EEG in localizing the epileptogenic zone: a study of the surgical resection outcome. Epilepsy Res. 108, 1581-1590. doi: 10.1016/j.eplepsyres.2014.08.016

Bagic, A. I., Knowlton, R. C., Rose, D. F., Ebersole, J. S., and ACMEGS Clinical Practice Guideline (CPG) Committee (2011). American clinical magnetoencephalography society clinical practice guideline 1: recording and analysis of spontaneous cerebral activity. J. Clin. Neurophysiol. 28, 348-354. doi: 10.1097/WNP.0b013e3182272fed

Baillet, S. (2017). Magnetoencephalography for brain electrophysiology and imaging. Nat. Neurosci. 20, 327-339. doi: 10.1038/nn.4504

Bouet, R., Jung, J., Delpuech, C., Ryvlin, P., Isnard, J., Guenot, M., et al. (2012). Towards source volume estimation of interictal spikes in focal epilepsy using magnetoencephalography. Neuroimage 59, 3955-3966. doi: 10.1016/j.neuroimage.2011.10.052 a large database of normal resting-state mapping should be established. With a temporally rich source of information on brain network dynamics, MEG was called upon to join in the Human Connectome Project (Larson-Prior et al., 2013). Three years later, Niso et al. (2015) published an open archive restingstate MEG data of 97 health participants, which mainly focus on the distributions of typical oscillation frequency bands (delta, theta, alpha, beta, and gamma). Interestingly, a previous study shown that coherent neural activity change in the beta band might be causally involved in epilepsy (Heers et al., 2014), suggesting an exciting practicability of this MEG normative repository in preoperative evaluation for individual epilepsy patient.

To sum up, with the development of cognitive neuroscience, our understanding of normal and abnormal functional mechanism of the brain will get better and better. Using advanced MEG analysis technology, objective biomarks to locate epileptic foci is likely to replace the traditional subjective judgments in the upcoming future.

\section{AUTHOR CONTRIBUTIONS}

QW, PT, and GL all contributed to the writing and revision of this manuscript.

\section{FUNDING}

This work was supported by the National Natural Science Foundation of China (81671285), the China Postdoctoral Science Foundation (2016M601066), the Capital Health Research and Development of Special (2016-1-8012), Beijing Municipal Science and Technology Commission (Z161100000516230, Z161100002616016).
Bressler, S. L., and Menon, V. (2010). Arge-scale brain networks in cognition: emerging methods and principles. Trends Cog. Sci. 14, 277-290. doi: 10.1016/j.tics.2010.04.004

Cataldi, M., Avoli, M., and Villers-Sidani, E. (2013). Resting state networks in temporal lobe epilepsy. Epilepsia 54, 2048-2059. doi: 10.1111/epi. 12400

Chowdhury, R. A., Lina, J. M., Kobayashi, E., and Grova, C. (2013). MEG source localization of spatially extended generators of epileptic activity: comparing entropic and hierarchical bayesian approaches. PLoS ONE 8:e55969. doi: 10.1371/journal.pone.0055969

Cohen, D. (1968). Magnetoencephalography: evidence of magnetic fields produced by alpha-rhythm currents. Science 161, 784-786. doi: $10.1126 /$ science.161.3843.784

Duncan, J. S., Winston, G. P., Koepp, M. J., and Ourselin, S. (2016). Brain imaging in the assessment for epilepsy surgery. Lancet Neurol. 15, 420-433. doi: 10.1016/S1474-4422(15)00383-X

Ebersole, J. S. (1997). Defining epileptogenic foci: past, present, future. J. Clin. Neurophysiol. 14, 470-483. doi: 10.1097/00004691-199711000-00003

Englot, D. J., Hinkley, L. B., Kort, N. S., Imber, B. S., Mizuiri, D., Honma, S. M., et al. (2015a). Global and regional functional connectivity maps of neural oscillations in focal epilepsy. Brain 138, 2249-2262. doi: 10.1093/brain/awv130

Englot, D. J., Nagarajan, S. S., Imber, B. S., Raygor, K. P., Honma, S. M., Mizuiri, D., et al. (2015b). Epileptogenic zone localization using magnetoencephalography predicts seizure freedom in epilepsy surgery. Epilepsia 56, 949-958. doi: $10.1111 /$ epi.13002 
Fisher, R. S., Van Emde Boas, W., Blume, W., Elger, C., Genton, P., Lee, P., et al. (2005). Response: definitions proposed by the international league against epilepsy (ILAE) and the international bureau for epilepsy (IBE). Epilepsia 46, 1701-1702. doi: 10.1111/j.1528-1167.2005.00273_4.x

Gonzalez-Martinez, J., Bulacio, J., Alexopoulos, A., Jehi, J., Bingaman, W., Najm, I., et al. (2013). Stereoelectroencephalography in the "difficult to localize" refractory focal epilepsy: early experience from a north american epilepsy center. Epilepsia 54, 323-330. doi: 10.1111/j.1528-1167.2012.03672.x

Grova, C., Aiguabella, M., Zelmann, R., Lina, J. M., Hall, J. A., Kobayashi, E., et al. (2016). Intracranial EEG potentials estimated from MEG sources: a new approach to correlate MEG and iEEG data in epilepsy. Hum. Brain Mapp. 37, 1661-1683. doi: 10.1002/hbm.23127

Haneef, Z., Lenartowicz, A., Yeh, H. J., Engel, J. Jr., and Stern, J. M. (2012). Effect of lateralized temporal lobe epilepsy on the default mode network. Epilepsy Behav. 25, 350-357. doi: 10.1016/j.yebeh.2012.07.019

Heers, M., Chowdhury, R. A., Hedrich, T., Dubeau, F., Hall, J. A., Lina, J. M., et al. (2016). Localization accuracy of distributed inverse solutions for electric and magnetic source imaging of interictal epileptic discharges in patients with focal epilepsy. Brain Topogr. 29, 162-181. doi: 10.1007/s10548-014-0423-1

Heers, M., Hedrich, T., An, D., Dubeau, F., Gotman, J., Grova, C., et al. (2014). Spatial correlation of hemodynamic changes related to interictal epileptic discharges with electric and magnetic source imaging. Hum. Brain Mapp. 35, 4396-4414. doi: 10.1002/hbm.22482

Heers, M., Rampp, S., Stefan, H., Urbach, H., Elger, C. E., von Lehe, M., et al. (2012). MEG-based identification of the epileptogenic zone in occult periinsular epilepsy. Seizure 21, 128-133. doi: 10.1016/j.seizure.2011.10.005

Jobst, B. C., and Cascino, G. D. (2015). Resective epilepsy surgery for drug-resistant focal epilepsy: a review. JAMA 313, 285-293. doi: 10.1001/jama.2014.17426

Knowlton, R. C., Razdan, S. N., Limdi, N., Elgavish, R. A., Killen, J., Blount, J., et al. (2009). Effect of epilepsy magnetic source imaging on intracranial electrode placement. Ann. Neurol. 65, 716-723. doi: 10.1002/ana.21660

Kobayashi, K., Yoshinaga, H., Ohtsuka, Y., and Gotman, J. (2005). Dipole modeling of epileptic spikes can be accurate or misleading. Epilepsia 46, 397-408. doi: 10.1111/j.0013-9580.2005.31404.x

Krishnan, B., Vlachos, I., Wang, Z. I., Mosher, J., Najm, I., Burgess, R., et al. (2015). Epileptic focus localization based on resting state interictal MEG recordings is feasible irrespective of the presence or absence of spikes. Clin. Neurophysiol. 126, 667-674. doi: 10.1016/j.clinph.2014.07.014

Larson-Prior, L. J., Oostenveld, R., Della Penna, S., Michalareas, G., Prior, F., Babajani-Feremi, A., et al. (2013). Adding dynamics to the human connectome project with MEG. Neuroimage 80, 190-201. doi: 10.1016/j.neuroimage.2013.05.056

Laufs, H. (2012). Functional imaging of seizures and epilepsy: evolution from zones to networks. Curr. Opin. Neurol. 25, 194-200. doi: 10.1097/WCO.0b013e3283515db9

Maccotta, L., He, B. J., Snyder, A. Z., Eisenman, L. N., Benzinger, T. L., Ances, B. M., et al. (2013). Impaired and facilitated functional networks in temporal lobe epilepsy. Neuroimage Clin. 2, 862-872. doi: 10.1016/j.nicl.2013.06.011

Malinowska, U., Badier, J. M., Gavaret, M., Bartolomei, F., Chauvel, P., and Bénar, C. G. (2014). Interictal networks in magnetoencephalography. Hum. Brain Mapp. 35, 2789-2805. doi: 10.1002/hbm.22367

Mikuni, N., Nagamine, T., Ikeda, A., Teradaa, K., Taki, W., Kimura, J. et al. (1997). Simultaneous recording of epileptiform discharges by MEG and subdural electrodes in temporal lobe epilepsy. Neuroimage 5, 298-306. doi: 10.1006/nimg.1997.0272

Mohamed, I. S., Gibbs, S. A., Robert, M., Bouthillier, A., Leroux, J.-M., and Nguyen, D. K. (2013). The utility of magnetoencephalography in the presurgical evaluation of refractory insular epilepsy. Epilepsia 54, 1950-1959. doi: 10.1111/epi.12376

Mosher, J. C., Lewis, P. S., and Leahy, R. M. (1992). Multiple dipole modeling and localization from spatio-temporal MEG data. IEEE Trans. Biomed Eng. 39, 541-557. doi: 10.1109/10.141192

Munsell, B. C., Wee, C. Y., Keller, S. S., Weberd, B., Elgerd, C., da Silvaa, A. T., et al. (2015). Evaluation of machine learning algorithms for treatment outcome prediction in patients with epilepsy based on structural connectome data. Neuroimage 118, 219-230. doi: 10.1016/j.neuroimage.2015 06.008

Murakami, H., Wang, Z. I., Marashly, A., Krishnan, B., Prayson, R. A., Kakisaka, Y., et al. (2016). Correlating magnetoencephalography to stereoelectroencephalography in patients undergoing epilepsy surgery. Brain 139:aww215. doi: 10.1093/brain/aww215

Niso, G., Rogers, C., Moreau, J. T., Moreaua, J. T., Chena, L.-Y., Madjarb, C., et al. (2015). Omega: the open MEG archive. Neuroimage 124(Pt B), 1182-1187. doi: 10.1016/j.neuroimage.2015.04.028

Oishi, M., Kameyama, S., Masuda, H., Masuda, H., Tohyama, J., Kanazawa, O., et al. (2006). Single and multiple clusters of magnetoencephalographic dipoles in neocortical epilepsy: significance in characterizing the epileptogenic zone. Epilepsia 47, 355-364. doi: 10.1111/j.1528-1167.2006.00428.x

Oishi, M., Otsubo, H., Kameyama, S., Morota, N., Masuda, H., Kitayama, M., et al. (2002). Epileptic spikes: magnetoencephalography versus simultaneous electrocorticography. Epilepsia 43, 1390-1395. doi: 10.1046/j.1528-1157.2002.10702.x

Salayev, K. A., Nakasato, N., Ishitobi, M., Shamoto, H., Kanno, A., and Iinuma K. (2006). Spike orientation may predict epileptogenic side across cerebral sulci containing the estimated equivalent dipole. Clin. Neurophysiol. 117, 1836-1843. doi: 10.1016/j.clinph.2006.05.003

Santiuste, M., Nowak, R., Russi, A., Arancon, T., Oliver, B., Ayats, E., Scheler, G., Graetz, G., et al. (2008). Simultaneous magnetoencephalography and intracranial EEG registration: technical and clinical aspects. J. Clin. Neurophysiol. 25, 331-339. doi: 10.1097/WNP.0b013e31818e7913

Shigeto, H., Morioka, T., Hisada, K., Nishio, S. Ishibashi, H., Kira, D.-I., et al. (2002). Feasibility and limitations of magnetoencephalographic detection of epileptic discharges: simultaneous recording of magnetic fields and electrocorticography. Neurol. Res. 24, 531-536. doi: 10.1179/016164102101200492

Tadel, F., Baillet, S., Mosher, J. C., Mosher, J. C., Pantazis, D., and Leahy, R. M., (2011). Brainstorm: a user-friendly application for MEG/EEG analysis. Comput. Intell. Neurosci. 2011, 879716. doi: 10.1155/2011/ 879716

Tanaka, N., Peters, J. M., Prohl, A. K., Takaya, S., Madsen, J. R., Bourgeois, B. F., et al. (2014). Clinical value of magnetoencephalographic spike propagation represented by spatiotemporal source analysis: correlation with surgical outcome. Epilepsy Res. 108, 280-288. doi: 10.1016/j.eplepsyres.2013. 11.006

Tao, J. X., Baldwin, M., Hawes-Ebersole, S., and Ebersole, J. S. (2007). Cortical substrates of scalp EEG epileptiform discharges. J. Clin. Neurophysiol. 24, 96-100. doi: 10.1097/WNP.0b013e31803ecdaf

Zerouali, Y., Pouliot, P., Robert, M., Mohamed, I., Bouthillier, A., Lesage, F., et al. (2016). Magnetoencephalographic signatures of insular epileptic spikes based on functional connectivity. Hum. Brain Mapp. 37, 3250-3261. doi: 10.1002/hbm.23238

Zhang, R., Wu, T., Wang, Y., Liu, H., Zou, Y., Liu, W., et al. (2011). Interictal magnetoencephalographic findings related with surgical outcomes in lesional and nonlesional neocortical epilepsy. Seizure 20, 692-700. doi: 10.1016/j.seizure.2011.06.021

Zhou, J., Huang, L. T., Zhai, F., Guan, Y, -G., Bao, M., Zhao, M., et al. (2017). The role of ROSA navigated intracranial electrode implantation technique on precise epileptogenic zone localization. Neuropsychiatry 7, 258-264. doi: 10.4172/Neuropsychiatry. 1000208

Conflict of Interest Statement: The authors declare that the research was conducted in the absence of any commercial or financial relationships that could be construed as a potential conflict of interest.

Copyright (c) 2017 Wang, Teng and Luan. This is an open-access article distributed under the terms of the Creative Commons Attribution License (CC BY). The use, distribution or reproduction in other forums is permitted, provided the original author(s) or licensor are credited and that the original publication in this journal is cited, in accordance with accepted academic practice. No use, distribution or reproduction is permitted which does not comply with these terms. 